\title{
Role of progressive muscle relaxation in preventing and alleviating of nausea and vomiting caused by chemotherapy among cancer patients: a systematic review of six randomized controlled trials
}

\author{
Xu Tian ${ }^{1}$, Ling-Li Xu ${ }^{2}$, Rong-Ying Tang ${ }^{2}$, Hui Chen ${ }^{1}$, Yuan-Ping Pi ${ }^{2}$, Wei-Qing Chen ${ }^{\text {* }}$ \\ 1 Department of Gastroenterology, Chongqing Key Laboratory of Translational Research for Cancer \\ Metastasis and Individualized Treatment, Chongqing University Cancer Hospital \& Chongqing Cancer \\ Institute \& Chongqing Cancer Hospital, Chongqing 400030, China; yxtx880919@hotmail.com \\ 2 Department of Nursing, Key Laboratory for Biorheological Science and Technology of Ministry of \\ Education (Chongqing University), Chongqing University Cancer Hospital \& Chongqing Cancer Institute \\ \& Chongqing Cancer Hospital, Chongqing 400030, China; cqzlyyhlb@163.com \\ * Correspondence: chenwq20140809@163.com; Tel.: +86-0-18222275023 (W.Q.C.)
}

\begin{abstract}
Background: Previous systematic review suggested a beneficial effect of progressive muscle relaxation (PMR) for cancer patients receiving chemotherapy. However, poor quality of eligible studies impaired the reliability and validity of findings. Moreover, additional potential studies with good quality published in Chinese language have been published recently. The aim of the present systematic review was to investigate the value of PMR training in preventing and alleviating nausea and vomiting caused by chemotherapy among cancer patients. (2) Methods: We assigned two independent investigators to search all potential studies in PubMed, Cochrane Controlled Register of Trial (CENTRAL), Cumulative Index to Nursing and Allied Health Literature (CINAHL), China Biomedical Literature database (CBM), China National Knowledge Infrastructure (CNKI), and Wanfang data. We used data extraction sheet extracted all essential information, and used the Cochrane risk of bias assessment tool to appraise the quality of eligible studies. Finally, we qualitatively summarized the results of all included studies. (3) Results: Six studies enrolling 288 patients were considered to meet our selection criteria finally. Of these 6 studies, three were labeled as moderate quality, and the remaining studies were low quality. All included studies consistently suggested that PMR has a positive impact on nausea and vomiting caused by chemotherapy among cancer patients especially alleviating the incidence, frequency and degree of delayed nausea and vomiting. (4) Conclusions: Independent studies indicated that PMR was a beneficial approach to prevent and alleviate nausea and vomiting caused by chemotherapy among cancer patients. However, further studies considering other types of primary tumors should be designed in order to increase the generality of PMR because studies included in the present systematic review mainly enrolled lung cancer and breast cancer.
\end{abstract}

Keywords: Cancer; Chemotherapy; Nausea; Vomiting; Progressive muscle relaxation

\section{Introduction}

According to the Global Cancer Statistics published in 2018, it is estimated that approximately 18.1 million new cancer cases and 9.6 million cancer deaths will occur in 2018[1]. Chemotherapy has been the decisive element of the multidisciplinary cancer treatment regime[2], and it is reported that chemotherapy was associated with prolonged survival and decreased cancer-related mortality and morbidity[3]. Nonetheless, a successful course of chemotherapy will cause significant various of serious side effects such as hair loss, loss of appetite, nausea, vomiting, temporary or permanent 
frigidity or impotence, as well as negative affects [4-6]. Of which, nausea and vomiting (CINV) is the most distressing one[7].

Serious nausea and vomiting not only lead to malnutrition, electrolyte disturbances, and dehydration[8], but also greatly impair functional status and the quality of life in cancer patients underwent chemotherapy[9]. It is noted that cancer patients suffering from distressing nausea and vomiting always interrupt chemotherapy regime[10]. However, refusal of or withdraw from the prescribed chemotherapy regime might be the major contributor to a reduction of survival time[11]. Hence, considerable efforts have been made towards order to effectively prevent and alleviate nausea and vomiting caused by chemotherapy. At present, use of antiemetics remains the cornerstone of managing nausea and vomiting related to chemotherapy, whereas prophylactic use of antiemetics are not applicable for all patients receving chemotherapy. More importantly, antiemetics will also be associated with several of undesirable side effects. Considering these issues, researchers and practitioners changed their attention from pharmacological treatment to non-pharmacological intervention. As the most common non-pharmacological option, progressive muscle relaxation (PMR) exercise has been widely applied to prevent and alleviate the toxic resulted from chemotherapy. Consequently, sporadic studies [12-17] were also performed to investigate the value of PMR for prevention and alleviation of nausea and vomiting caused by chemotherapy.

Although a previous systematic review has explored the role of PMR among cancer patients receiving chemotherapy[18]. However, in this systematic review, Pelekasis and colleagues evaluated several outcomes such as nausea, vomiting, anxiety, fatigue, sleep quality and cancer discomfort[18]. That is to say, nausea and vomiting were not be fully focused. Moreover, most important is the fact that some studies published in the Chinese language were not enrolled [14,15,17]. It is important to note that, however, it a critical characteristic of a systematic review to capture all potential studies worldwide[19]. So, is critical to design a systematic review to fully investigate the efficacy and safety of PMR for prevention and alleviation of nausea and vomiting induced by chemotherapy among cancer patients.

\section{Results}

\subsection{Result of identification}

We delineated the process of searching and screening citations in Figure 1. We captured 211 records after initially searched all targeted databases, and 119 unique records were remained after removing duplicates using the literature management software EndNote X7. We further excluded 79 records after carefully checking the title and abstract of each item, and thus 40 items were included to be checked in full-text. Finally, 6 studies [12-17] enrolling 288 patients were deemed to meet our selection criteria after excluding from 36 studies due to several reasons including unrelated to topic and ineligible patients and intervention regimes. 


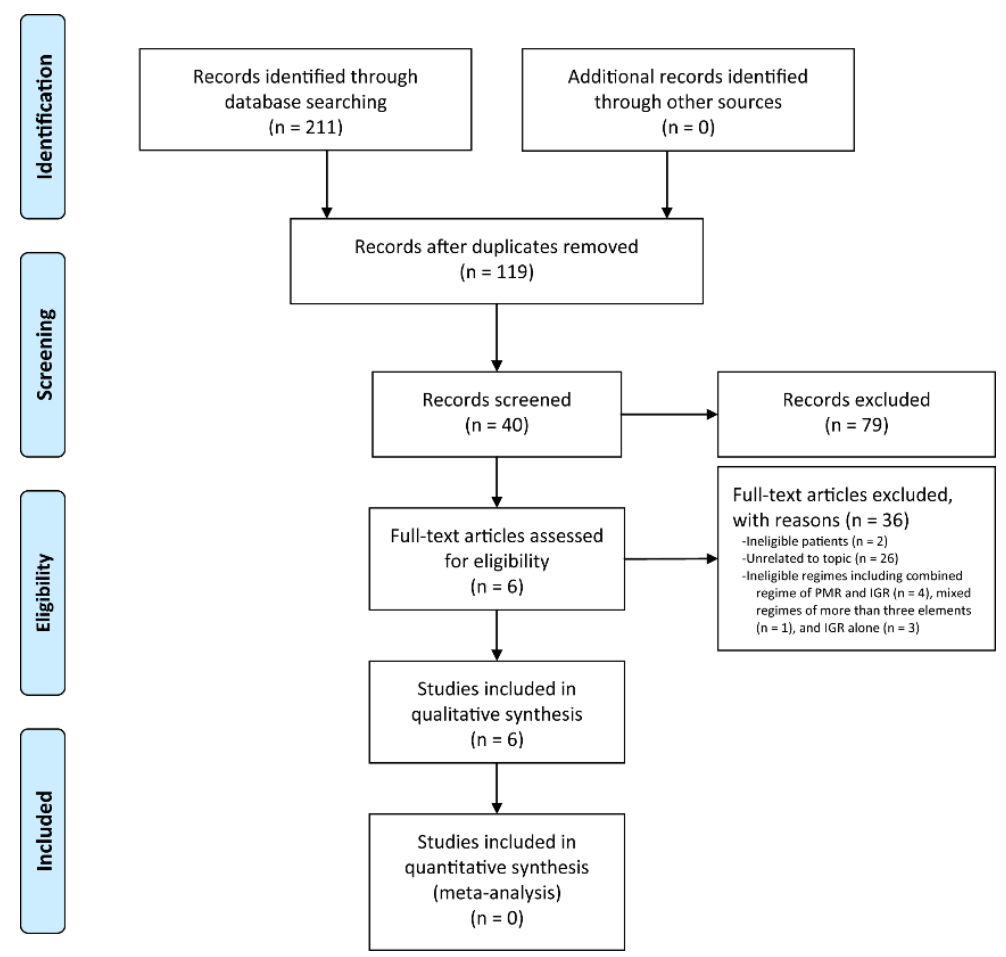

Figure 1. Flow diagram of searching and selecting citations. PMR, progressive muscle relaxation; IGR, image-guided relaxation.

\subsection{Characteristics of included studies}

We documented the basic characteristics of all eligible studies in Table 1. Of these 6 eligible studies, 4[14-17] were were performed in China and remaining two $[12,13]$ were completed in Japan. The sample size of individual study was ranging from 8 to 100 with a median of 64 . Two studies $[15,17]$ enrolled patients with lung cancer, two $[14,16]$ enrolled breast cancer patients, and two $[12,13]$ included mixed cancer patients. Five studies $[12-15,17]$ reported the average adherence rate of ranging from $85.8 \%$ to $100 \%$ except for one[16] in which this index was not included. Five measures including Common Terminology Criteria for Adverse Events (CTCAE) [17], Multinational Association of Supportive Care in Cancer Antiemesis Tool (MASCC-AT) [14,15], Index of Nausea and Vomiting-Form 2 (INV-2)[13], Morrow of Nausea and Emesis (MANE)[12], and Rotterdam Symptom Scale (RSS)[16] were used to evaluate the outcomes in all eligible studies.

\subsection{Risk of bias of all eligible studies}

The risk of bias for each study was presented in Figure 2. Of included 6 studies, three $[14,15,17]$ adopted a random number table to generate random sequence, two[12,13] did not report the details of randomization, and one[16] assigned patients based on an order of admission. One [16] study did not conceal the random sequence. Three studies $[12,13,16]$ did not blinded investigators and patients. Two studies $[12,13]$ were identified as have high risk of attrition bias. Moreover, two studies $[12,13]$ were also judged to be high risk in terms of other risk sources because pre-/post design was adopted. So, three studies $[12,13,16]$ were labeled as high risk of bias, and remaining three $[14,15,17]$ were labeled as unclear risk of bias. Finally, we rated the whole quality of all included studies to be low level due to a half of studies were labeled as high risk of bias. 
103 Table 1. Basic characteristics of included 6 studies

\begin{tabular}{|c|c|c|c|c|c|c|c|c|c|c|c|}
\hline \multirow{2}{*}{ Study } & \multirow{2}{*}{ Country } & \multirow{2}{*}{$\begin{array}{l}\text { Sample size } \\
\text { (CG/IG) }\end{array}$} & \multicolumn{2}{|c|}{$\begin{array}{c}\text { Sex } \\
\text { (male/female) }\end{array}$} & \multirow{2}{*}{$\begin{array}{c}\text { Age } \\
(\mathrm{CG} / \mathrm{IG})\end{array}$} & \multirow{2}{*}{$\begin{array}{l}\text { Primary } \\
\text { tumor }\end{array}$} & \multirow{2}{*}{ Details of PMR exercise } & \multirow{2}{*}{$\begin{array}{l}\text { Duration of } \\
\text { follow-up }\end{array}$} & \multirow{2}{*}{$\begin{array}{c}\text { Average } \\
\text { adherence } \\
\text { rate }\end{array}$} & \multirow{2}{*}{ Measures used } & \multirow{2}{*}{ Main findings } \\
\hline & & & CG & IG & & & & & & & \\
\hline $\begin{array}{l}\text { Zhang, et } \\
\text { al., } 2017\end{array}$ & China & $50(25 / 25)$ & $16 / 9$ & $14 / 11$ & $\begin{array}{l}(54.7 \pm 5.8) / \\
56.1 \pm 6.4)\end{array}$ & $\begin{array}{l}\text { Lung } \\
\text { cancer }\end{array}$ & $\begin{array}{c}\text { Once a day, lasting for } 3 \text { weeks, } \\
\text { duration of per session was } \\
\text { unclear. }\end{array}$ & $\begin{array}{c}3 \text { weeks after } \\
\text { treatment }\end{array}$ & $100 \%$ & CTCAE & $\begin{array}{l}\text { PMR reduced the incidecne of } \\
\text { nausea and vomiting. }\end{array}$ \\
\hline $\begin{array}{l}\text { Li, et al., } \\
2019\end{array}$ & China & $68(34 / 34)$ & \multicolumn{2}{|c|}{ All were femal } & $\begin{array}{c}(47.32 \pm 7.45) /(4 \\
8.97 \pm 7.78)\end{array}$ & $\begin{array}{l}\text { Breast } \\
\text { cancer }\end{array}$ & $\begin{array}{l}\text { Twice a day, } 30 \text { minutes per } \\
\text { session, lasting for } 4 \text { days. }\end{array}$ & $\begin{array}{c}2 \text { repeated } \\
\text { measures from } \\
\text { day } 1 \text { to day } 4 \\
\text { after start of } \\
\text { chemotherapy }\end{array}$ & $100 \%$ & MASCC-AT & $\begin{array}{l}\text { PMR reduced the incidence } \\
\text { and frequency of delayed } \\
\text { vomiting, and allievated } \\
\text { delayed nausea. }\end{array}$ \\
\hline $\begin{array}{l}\text { Luo, et } \\
\text { al., } 2016\end{array}$ & China & $72(36 / 36)$ & \multicolumn{2}{|c|}{$52 / 20$} & $21-72(57.0)$ & $\begin{array}{l}\text { Lung } \\
\text { cancer }\end{array}$ & $\begin{array}{l}\text { Once a day, } 25 \text { minutes per } \\
\text { session, lasting for } 7 \text { days. }\end{array}$ & $\begin{array}{l}7 \text { days after } \\
\text { treatment }\end{array}$ & $100 \%$ & MASCC-AT & $\begin{array}{l}\text { PMR reduced the number of } \\
\text { acute vomiting, and the } \\
\text { frequency and number of } \\
\text { delayed vomiting, and } \\
\text { allievated degree of delayed } \\
\text { nausea. }\end{array}$ \\
\hline $\begin{array}{l}\text { Arakawa, } \\
\text { et al., } \\
1997\end{array}$ & Japan & $60(30 / 30)$ & $20 / 10$ & $16 / 14$ & $\begin{array}{c}(57.7 \pm 12.4) /(56 . \\
5 \pm 10.5)\end{array}$ & $\begin{array}{l}\text { Mixed } \\
\text { tumors }\end{array}$ & $\begin{array}{l}\text { Twice a day, } 25 \text { minutes per } \\
\text { session, lasting for } 72 \text { hours. }\end{array}$ & $\begin{array}{l}72 \text { hours after } \\
\text { treatment }\end{array}$ & $85.8 \%$ & INV-2 & $\begin{array}{l}\text { PMR reduced the scores of } \\
\text { nausea but did not vomiting. }\end{array}$ \\
\hline $\begin{array}{l}\text { Arakawa, } \\
\text { et al., } \\
1995\end{array}$ & Japan & $8(4 / 4)$ & $3 / 1$ & $1 / 3$ & $\begin{array}{c}(57.5 \pm 15.2) /(48 . \\
8 \pm 18.9)\end{array}$ & $\begin{array}{l}\text { Mixed } \\
\text { tumors }\end{array}$ & $\begin{array}{c}\text { Twice a day, } 15 \text { minutes per } \\
\text { session, duration of the whole } \\
\text { intervention was unclear. }\end{array}$ & n.r. & $98 \%$ & MANE & $\begin{array}{l}\text { PMR decreased the frequency } \\
\text { of posttreatment. }\end{array}$ \\
\hline $\begin{array}{l}\text { Song, et } \\
\text { al., } 2013\end{array}$ & China & $100(50 / 50)$ & All we & femal & $\begin{array}{c}25-70 \\
(43.6 \pm 12.7)\end{array}$ & $\begin{array}{l}\text { Breast } \\
\text { cancer }\end{array}$ & $\begin{array}{l}\text { Lasting for full duration of } \\
\text { chemotherapy, frequency and } \\
\text { duration of per session were } \\
\text { unclear. }\end{array}$ & $\begin{array}{c}\text { After } \\
\text { chemotherapy }\end{array}$ & n.r. & RSS & $\begin{array}{l}\text { PMR reduced the incidence of } \\
\text { nausea. }\end{array}$ \\
\hline
\end{tabular}

CG, control group; IG, intervention group; CTCAE, Common Terminology Criteria for Adverse Events; MASCC-AT, Multinational Association of Supportive Care in Cancer Antiemesis Tool; INV-2, Rhodes Index of Nausea and Vomiting-Form 2; MANE, Morrow of Nausea and Emesis; RSS, Rotterdam Symptom Scale. n.r. not reported. 


\subsection{Qualitative summaries of all ieligible studies}

In 1995, Arakawa and colleagues performed a pilot trial, in which 8 patients with various primary tumors were entered, to examine the effects of PMR on CINV [12]. Patients in control group were assigned to receive standard treatment, and patients assigned in intervention group were instructed to practice PMR program twice a day, which includes tense/release of 16 muscle groups and deep breathing (for a total of 15 minutes), however duration of the whole intervention was not introduced clearly. Researchers assessed CINV before and after chemotherapy with the MANE tool. After completed this study, an average adherence rate of $98 \%$ was reported. This study indicated that the PMR program decreased the frequency and duration of chemotherapy induced nausea, however did not affect chemotherapy related vomiting.

Arakawa and colleagues, in 1997, performed another study to further examine the effects of PMR program on nausea and vomiting in cancer patients receiving chemotherapy [13]. In this study, 60 Japanese patients with mixed tumors who were hospitalized in a cancer centre receiving chemotherapy were recruited and further assigned randomly to PMR and control groups. Patients in the intervention group were asked to exercise PMR program twice a day for 72 hours, and patients in the control group received route nursing care in order to obtain the equal placebo effect to the intervention group. Anticipated outcome was evaluated using the Rhodes Index of Nausea and Vomiting-Form 2 (INV-2) one week before initiation of their initial course and 72 hours after receiving the standard chemotherapy treatment. This study reported an average adherence rate of $85.8 \%$. Although the total scores of INV-2 used to measure nausea and vomiting were considerably reduced, subscale analyses indicated that vomiting was not significantly decreased, possibly due to the extremely low incidence in both groups.

In 2013, Song and colleagues [16] investigate the potential of PMR in managing nausea and vomiting related to chemotherapy through performing a randomized controlled trial, in which 100 patients with breast cancer who were treated with chemotherapy were enrolled. Eligible patients were allocated to the PMR program group and control group according to the order of admission. This study used the Rotterdam Symptom Scale (RSS) to record chemotherapy-related symptoms, in which nausea was also evaluated. Unfortunately, the adherence rate was not listed in this study. Finally, researchers found that the score of nausea was significantly reduced in the PMR program group after exercising this technique.

In 2016, Luo and colleagues [15] carried out a randomized controlled trial to test the effect of PMR on nausea and vomiting related to chemotherapy. In this trial, a total of 72 lung cancer patients were divided equally into PMR program and control groups acting in accordance with a random number table. Patients in the control group were intravenously injected with tostron or palonosetron in order to prevent CINV at 30 min before carrying out chemotherapy. Patients assigned to the PMR group were instructed to exercise PMR program once a day for 7 days, however duration of the entire intervention was not reported clearly. CINV was measured with the Multinational Association of Supportive Care in Cancer Antiemesis (MASCC) tool 7 days after receiving chemotherapy. All patients completed this study. Results suggested that PMR program significantly decreased the number of acute vomiting, the frequency and number of delayed vomiting and level of delayed nausea.

Zhang and colleagues [17] performed a trial enrolling 50 patients with lung cancer to examine the effect of PMR intervention on nausea and vomiting resulted from chemotherapy in 2017. In each group, 25 patients were deemed to receive corresponding regime. Patients in the control group were treated using route nursing care, and patients in the intervention group were treated with PMR program once a day for 3 weeks. Nausea and vomiting were recorded using the Common Terminology Criteria for Adverse Events (CTCAE) tool which divided nausea and vomiting into 
five degrees and higher degree indicates a higher level of adverse effect. During the entire study, all patients completed corresponding intervention regimes. Three weeks after intervention, authors compared the incidence of nausea and vomiting between control and intervention groups. Finally, results suggested that the incidence of nausea and vomiting in PMR program group was less than that in control group ( $16 \%$ vs. $44 \%)$, with significant difference.

In 2019, Li and colleagues [14] recruited 68 breast cancer patients underwent chemotherapy to explore the effect of PMR exercise on nausea and vomiting related to chemotherapy. Eligible patients were randomly assigned into the control and intervention groups based on a random number table. Patients in the control group were intravenously injected with tropisetron and dexamethasone for prophylactic antiemetic, and patients in the intervention group were arranged to complete PMR exercise twice a day for 4 days. The Multinational Association of Supportive Care in Cancer Antiemesis (MASCC) tool was used to assess the nausea and vomiting at 24 hours and 4 days after chemotherapy. All eligible patients adhered to the corresponding to the regime during the entire study. Finally, this study indicated that PMR exercise decreased the incidence and frequency of delayed vomiting and relived the degree of delayed nausea, with significantly different.

\section{Discussion}

Cancer has become the major cause of causing mortality and morbidity worldwide [1]. Although chemotherapy is universally acknowledged being associated with prolonged survival time and improved quality of life, it will cause several of toxic effects especially nausea and vomiting [7]. Patients will experience distressing nausea and vomiting when initiation of chemotherapy stimulates emetic center through triggering various neurologic pathways [8], especially stimulation of the chemoreceptor trigger zone (CTZ) [22]; other inputs to the vomiting center play a lesser role [23].

Efforts have been made to develop pharmacological interventions in order to effectively control or alleviate nausea and vomiting caused by chemotherapy among cancer patients, however approximately $60 \%$ of patients who were prophylactically treated with antiemetics continue to suffer from nausea[24]. So, attention on chemotherapy induced nausea and vomiting has been changed from pharmacological interventions to non-pharmachological regimes [25], of which progressive muscle relaxation (PMRT) is the most widely option [26]. The results of the present systematic review indicated that several independent studies have reported a significant beneficial effect of PMR training on the nausea and vomiting related to chemotherapy as measured by relevant tools.

To date, a few piecemeal studies reported the role of PMR training in preventing and treating chemotherapy induced nausea and vomiting among cancer patients, however findings from most of the studies consistently suggested that PMR has positive impact on this condition [2], which were consistent with our findings. For example, Burish and Tope[27] performed a review to summarize the applications of PMR in cancer patients, and drawn four key conclusions, of which two were related to nausea and vomiting as following: (a) PMRT is effective in reducing toxic effects of chemotherapy such as conditioned nausea and vomiting; and (b) if taught before the initiation of chemotherapy, relaxation therapy including PMR exercise has the potential of preventing or considerably delaying the onset of conditioned responses if it would be performed before initiating chemotherapy regimes. Moreover, Morrow and colleagues [28] also found that progressive muscle relaxation training effectively prevented and decreased the frequency of post-chemotherapy nausea and vomiting. This finding also verified the effect of PMR training in preventing and relieving nausea and vomiting related to chemotherapy. More importantly, Pelekasi and colleagues [18] carried out a systematic review to comprehensively evaluate the value of PMR exercise as an adjunctive regime for cancer patients receiving chemotherapy. In this systematic review, 3 of five included studies which reported nausea and vomiting also generated a promising conclusion supporting positive application of PMR in cancer patients underwent chemotherapy. In our systematic review, we added three additional randomized controlled trials $[14,15,17]$ with moderate quality to obtain similar finding. 
Although the sample size of individual study included in the present systematic review was varied from one to other, all studies generated consistent conclusion of supporting the value of PMR exercise. And thus, we strongly believe that this difference of these studies will not impair the reliability of our findings. Moreover, despite the fact that three additional studies added to our systematic review were performed in China, the quality of each study was rated to be a moderate level. We also found that, in the present systematic review, five measurement tools were adopted to record outcome, and the psychometric characteristics of some tools were not tested. It is important to note that, however, these studies found the same results through the application of different measurement tools, and consequently, the internal validity of our systematic review would be enhanced [29].

Certainly, other aspects may have impact on the reliability and validity of our findings should be discussed. Firstly, we noted that, in the present systematic review, included studies only considered breast cancer[14,16], lung cancer[15,17] and mixed tumors[12,13], Thus, it is essential to design further studies enrolling patients with different primary tumors in order to increase the generalizability of the study results on patients with other types of cancer. Secondly, all included studies in the present systematic review were carried out in Asian countries, which downgraded the external validity of our findings. Thirdly, although all included studies instructed patients assigned to the intervention group to practice PMR training, the details of each program were different especially timeframe of each session and duration of the whole intervention. So, we strongly suggested to standard the contents and timeframe of PMR training program [29]. Forthly, we captured additional three studies with relatively high quality through searching Chinese databases, however three studies analyzed in published systematic review were low quality. After carefully reviewing the details of risk of bias of these three studies, we found that many factors caused high risk of bias was method of blinding investigators, participants and assessors and of generating a random sequence. Hence, further study should address these issues for the purpose of increasing the level of evidence.

\section{Materials and Methods}

We designed and performed this systematic review in accordance with the recommendations suggested by the Cochrane Collaboration (CC) [19]. The preferred items for systematic review and meta-analysis (PRISMA) statement were adopted to guide us to report all results [20]. Ethic approval and informed consent were not required because we performed all statistical analyses and summarized all evidence based on published data.

\subsection{Selection criteria}

Randomised controlled trials published in peer-reviewed academic journals, considering PMR training program, enrolling cancer patients treated with chemotherapy were regarded as the inclusion criteria. Studies combined PMR exercies with other regimes, such as image guided (IG) relaxation or physical activity was excluded. Moreover, we excluded superiority trials because this design was beyond the objective of our systematic review.

The inclusion and exclusion criteria evaluation were carried out by X.T. and W.-Q.C. All potential conflicts regarding selection of studies were resolved by consulting a third senior investigator (W.-Q.C).

\subsection{Identification of citations}

A comprehensive search, which was completed by two independent investigators, was performed in several databases including PubMed, Cochrane Controlled Register of Trial (CENTRAL), Cumulative Index to Nursing and Allied Health Literature (CINAHL), China Biomedical Literature database (CBM), China National Knowledge Infrastructure (CNKI), and Wanfang data. Search time was limited from their inception to 30 May 2019. Moreover, we updated the search results per week. We constructed search algorithm based on a combination of medical subject heading (MeSH) and text words. The basic search string was the following: (Progressive Muscle Relaxation) AND (cancer OR 
oncology OR chemotherapy). All search algorithms were modified acting in accordance with the unique characteristics of each database. We also hand checked the bibliographies of topic-related reviews and eligible studies in order to incorporate any potential studies. We finished the search in accordance with the principles listed in the PRISMA statement [20]. Finally, we used the EndNote to store all captured items. The principle of consulting a third senior investigator was introduced to solve any divergency of search.

\subsection{Data extraction}

In this systematic review, all essential data including leading author, publication year, country, sample size, sex and age of patients, primary tumor, details of regimes, duration of follow-up, average adherence rate, measurement tool for outcome, and main findings were extracted by two independent investigators with a standard data extraction sheet. The corresponding author was contacted when sufficient information cannot be obtained. Any divergency of data extraction were solved through consulting a third senior investigator.

\subsection{Quality assessment of eligible study}

The quality of each eligible study was determined based on assessment result of the risk of bias, which was appraised by using the Cochrane risk of bias assessment tool [21] from the following six domains [19]: randomization, allocation, blind, incomplete data, selectively reported and other bias sources. A study would be labeled as 'low', 'unclear', or 'high' risk of bias [15] according to the match level between actual information and criteria. Any divergency at risk of bias were solved through a third senior investigator. Finally, the overall quality of each study would be graded as minimal, moderate or high according to the result of labelling of the risk of bias.

\section{Conclusions}

Independent trials with relatively good quality indicated that the PMR exercise is an efficient option to decrease the nausea and vomiting caused by chemotherapy. However, a major limitation in the present systematic review is that all included studies were performed in Asian countries, so more studies should be developed in order to determine the value of PMR exercise in other countries and regions. It must be noted that, moreover, all included studies in our systematic review were insufficient, so study with large scale should be designed when one expects to investigate the role of PMR exercise in further primary tumours.

Author Contributions: X.T. and W.Q.C. conceived and designed this study. X.T., L.L.X. and H.C. participated in study selection, data extraction and quality evaluation. X.T. and R.Y.T. summairzed the results of all included studies. X.T. and R.Y.T. prepared the draft. X.T. and W.Q.C. critically revised the whole manuscript. All authors approved the final version for submission.

Funding: This research received no external funding.

Acknowledgments: We would like to express our warm appreciation to all authors who designed and performed studies which were included in the present study.

Conflicts of Interest: The authors declare no conflict of interest.

\section{References}

1. Bray, F.; Ferlay, J.; Soerjomataram, I.; Siegel, R.L.; Torre, L.A.; Jemal, A. Global cancer statistics 2018: GLOBOCAN estimates of incidence and mortality worldwide for 36 cancers in 185 countries. CA Cancer J Clin 2018, 68, 394-424, doi:10.3322/caac.21492.

2. Cheung, Y.L.; Molassiotis, A.; Chang, A.M. A pilot study on the effect of progressive muscle relaxation training of patients after stoma surgery. Eur J Cancer Care (Engl) 2001, 10, 107-114. 
295 3. DeVita, V.T., Jr.; Chu, E. A history of cancer chemotherapy. Cancer Res 2008, 68, 8643-8653, doi:10.1158/0008296 5472.CAN-07-6611.

297 4. Andrykowski, M.A.; Jacobsen, P.B.; Marks, E.; Gorfinkle, K.; Hakes, T.B.; Kaufman, R.J.; Currie, V.E.; Holland, J.C.; 298 Redd, W.H. Prevalence, predictors, and course of anticipatory nausea in women receiving adjuvant chemotherapy for breast cancer. Cancer 1988, 62, 2607-2613.

$300 \quad$ 5. Burish, T.G.; Redd, W.H. Symptom control in psychosocial oncology. Cancer 1994, 74, 1438-1444.

301 6. Kvale, G.; Hugdahl, K.; Asbjornsen, A.; Rosengren, B.; Lote, K.; Nordby, H. Anticipatory nausea and vomiting in 302 cancer patients. J Consult Clin Psychol 1991, 59, 894-898.

303 7. Coates, A.; Abraham, S.; Kaye, S.B.; Sowerbutts, T.; Frewin, C.; Fox, R.M.; Tattersall, M.H. On the receiving end-304 patient perception of the side-effects of cancer chemotherapy. European journal of cancer \& clinical oncology 1983, 305 19, 203-208.

306 8. Furue, H. Antiemetics and its clinical evaluations. Gan to kagaku ryoho. Cancer \& chemotherapy 1992, 19, $294-301$. 307 9. Charalambous, A.; Giannakopoulou, M.; Bozas, E.; Marcou, Y.; Kitsios, P.; Paikousis, L. Guided Imagery And Progressive Muscle Relaxation as a Cluster of Symptoms Management Intervention in Patients Receiving Chemotherapy: A Randomized Control Trial. PLoS One 2016, 11, e0156911, doi:10.1371/journal.pone.0156911.

Pervan, V. Practical aspects of dealing with cancer therapy-induced nausea and vomiting. Semin Oncol Nurs 1990, 6, 3-5.

312 11. Die Trill, M. Anxiety and sleep disorders in cancer patients. EJC supplements : EJC : official journal of EORTC, European Organization for Research and Treatment of Cancer ... [et al.] 2013, 11, 216-224, doi:10.1016/j.ejcsup.2013.07.009. Arakawa, S. Use of relaxation to reduce side effects of chemotherapy in Japanese patients; 1995; Vol. 18, pp. 60-66. Arakawa, S. Relaxation to reduce nausea, vomiting, and anxiety induced by chemotherapy in Japanese patients. Cancer nursing 1997, 20, 342-349. Li, N.; Zhong, Y.P.; Sun, S.R. Efficacy of progressive muscle relaxation training on prevention of chemotherapyinduced nausea and vomiting in patients with breast cancer. Shanghai Nursing 2019, 19, 10-13. Luo, D.; Wang, Y.W.; Liu, H.P. Effect of relaxation training on acute and delayed nausea and vomiting induced by cisplatin in lung cancer patients. Journal of Nursing Science 2016, 31, 29-31. Song, Q.H.; Xu, R.M.; Zhang, Q.H.; Ma, M.; Zhao, X.P. Relaxation training during chemotherapy for breast cancer improves mental health and lessens adverse events. International journal of clinical and experimental medicine 2013, 6, 979-984.

Zhang, L.J.; Cai, S.; Zhao, H.; Liang, Y.T.; Ye, M.L.; Chen, C. Study on effects of progressive muscle relaxation training on nausea and vomiting, cancer related fatigue and negative emotion of patients with lung cancer undergoing chemotherapy. Chinese General Practice Nursing 2017, 15, 3338-3341.

Pelekasis, P.; Matsouka, I.; Koumarianou, A. Progressive muscle relaxation as a supportive intervention for cancer patients undergoing chemotherapy: A systematic review. Palliat Support Care 2017, 15, 465-473, doi:10.1017/S1478951516000870. Schmiedebergs Archiv für experimentelle Pathologie und Pharmakologie 2008, 5, S38. 